\title{
SOME LIMITS OF BOOLEAN ALGEBRAS
}

\section{FRANKLIN HAIMO}

1. Introduction. Inverse systems of topological spaces and direct and inverse systems of Abelian groups and resulting applications thereof to algebraic topology have been studied intensively, and the techniques employed are now standard [2]. (Numbers in brackets refer to the bibliography at the end of the paper.) We shall apply some of these ideas to the treatment of sets of Boolean algebras in order to obtain, in the inverse case, a representation, and, in both cases, some information about the ideals and about the decompositions of certain algebras. For instance, it is well known [1] that the cardinal product $B^{*}$ of a set $\left\{B_{\alpha}\right\}$ of Boolean algebras, indexed by a class $\mathrm{A}$, is also a Boolean algebra. Moreover, if each $B_{\alpha}$ is m-complete for some fixed cardinal $\mathfrak{m}[3]$, then $B^{*}$ is $\mathrm{m}$-complete. When $\mathrm{A}$ is a set partly ordered by $\leqq$ and directed thereby, and when the $B_{\alpha}$ form an inverse system (to be defined below), we shall show that the resulting inverse limit set is a Boolean algebra, a subalgebra of $B^{*}$, and a similar result will hold in the direct case, at least in part. ${ }^{1}$ For each nonfinite Boolean algebra $B$, there will be constructed a nontrivial inverse limit representation of $B$, the inverse limit of all principal ideals [3] of $B$ (with repetitions). In the direct case, ideals in the limit algebra (and the homomorphic algebras which they generate) will turn out to be direct limits of like objects. Direct decomposition into ideals of the direct limit takes place (it will be seen) if there is a similar decomposition in each $B_{\alpha}$.

Following a suggestion of the referee, we shall investigate the underlying Stone representation spaces of the algebras involved. It turns out that spaces associated with an inverse limit of algebras have a direct limit which is a Stone subspace of the Stone space of the inverse limit. On the other hand, the spaces associated with a direct limit of algebras have an inverse limit Stone space which is homeomorphic to the Stone space of the direct limit.

A Boolean algebra $B$ is defined to be a complemented distributive lattice [1] with partial ordering relation $\leqq$. Complements are denoted by primes, and the cup $(\cup)$-cap $(\cap)$ notation of $[1]$ is used practically without modification.

Presented to the International Congress of Mathematicians, September 1, 1950; received by the editors May $24,1950$.

1 See S. Bochner. Proc. Nat. Acad. Sci. U.S.A. vol. 36 (1950) pp. 439-443 
2. The inverse limit. Let $\mathrm{A}$ be a partly ordered set with order relation $\leqq$ which directs $\mathrm{A}$ in that $\alpha, \beta \in \mathrm{A}$ imply the existence of $\gamma \in \mathrm{A}$ with $\alpha, \beta \leqq \gamma$. Let $\left\{B_{\alpha}\right\}$ be a set of Boolean algebras indexed by $\mathbf{A}$. For each inequality $\beta \leqq \alpha(\alpha, \beta \in A)$, suppose that there exists a lattice homomorphism [1] $\Pi_{\beta \alpha}$ of $B_{\alpha}$ into $B_{\beta}$ in such a way that (1) if $I_{\beta}, 0_{\beta}\left(I_{\alpha}, 0_{\alpha}\right)$ are the supremum and infimum of $B_{\beta}\left(B_{\alpha}\right)$, then $\Pi_{\beta \alpha} I_{\alpha}$ $=I_{\beta}$, and $\Pi_{\beta \alpha} 0_{\alpha}=0_{\beta} ;$ and (2) $\gamma \leqq \beta \leqq \alpha(\gamma, \beta, \alpha \in A)$ imply $\Pi_{\gamma \alpha}$ $=\Pi_{\gamma \beta} \Pi_{\beta \alpha}$. We also require that $\Pi_{\alpha \alpha}$ be the identity mapping of $B_{\alpha}$ onto $B_{\alpha}$. Such a set of Boolean algebras and corresponding mappings will be called an inverse system (of Boolean algebras). Let $B^{*}$ be the cardinal product of the $B_{\alpha}$. Let $x \in B^{*}$ have component $x_{\alpha} \in B_{\alpha}$. For $x, y \in B^{*}, x \leqq y$ means that $x_{\alpha} \leqq y_{\alpha}$ for every $\alpha \in \mathrm{A}$. Let a subset $B^{i}$ of $B^{*}$ be defined as follows: $x \in B^{i}$ if $x \in B^{*}$ and if, for each inequality $\beta \leqq \alpha, \Pi_{\beta \alpha} x_{\alpha}=x_{\beta}$. $B^{i}$ has a hereditary partial order from $B^{*}$. The element $I=\left\{I_{\alpha}\right\} \in B^{i}$ is the supremum of $B^{i}$, and $0=\left\{0_{\alpha}\right\}$ is the infimum. It is trivial to show that under the ordering hereditary from $B^{*}, B^{i}$ is a Boolean algebra, a subalgebra of $B^{*}$. We might call $B^{i}$ the inverse limit of the factors $B_{\alpha}$ and write $B^{i}=\lim _{\leftarrow} B_{\alpha}$.

Let $\mathfrak{m}$ be a fixed infinite cardinal. Let $\left\{B_{\alpha}\right\}$ be an inverse system of Boolean algebras with mappings $\Pi_{\beta \alpha}$ and inverse limit $B^{i}$. Let each $\Pi_{\beta \alpha}$ have the property that if $x_{\alpha} \in B_{\alpha}$ is the least upper bound (dually, greatest lower bound) of the elements of a subset $S$ of $B_{\alpha}$, where power $S \leqq \mathfrak{m}$, then $\Pi_{\beta \alpha} x_{\alpha}=$ l.u.b. (g.l.b.) of the elements of $\Pi_{\beta \alpha} S$. Let such a set be called an m-potent inverse system.

TheOREM 1. Let $\left\{B_{\alpha}\right\}$ be an m-potent inverse system of Boolean algebras with inverse limit $B^{i}$. Let $\Gamma$ be a cofinal subset of $\mathrm{A}$ such that for $\gamma \in \Gamma, B_{\gamma}$ is m-complete. Then $B^{i}$ is an $\mathrm{m}$-complete Boolean algebra.

Proof. Let $\left\{x^{\sigma}\right\}$ be a class $S \subset B^{i}$, where $\sigma \in \Sigma$, an index class of power less than or equal to $\mathrm{m}$. For given $\alpha \in \mathrm{A}$, there exists $\gamma \in \Gamma$ such that $\alpha \leqq \gamma$, and $\Pi_{\alpha \gamma} x_{\gamma}^{\sigma}=x_{\alpha}^{\sigma}$. Let $x_{\gamma} \in B_{\gamma}$ be $\mathrm{V}_{\sigma} x_{\gamma}^{\sigma}$ (the 1.u.b. of the $\left.x_{\gamma}^{\sigma}\right)$. Then $x_{\alpha}=\Pi_{\alpha \gamma} x_{\gamma}=\mathrm{V}_{\sigma} x_{\alpha}^{\sigma}$, by the hypothesis on the $\Pi_{\alpha \gamma}$. Define $x \in B^{*}$ by $x=\left\{x_{\alpha}\right\}$. For each inequality $\beta \leqq \alpha$, there exists $\delta \in \Gamma$ with $\beta \leqq \alpha \leqq \delta$, and $\Pi_{\alpha \delta} x_{\delta}=x_{\alpha}, \Pi_{\beta \delta} x_{\delta}=x_{\beta}$. Hence $\Pi_{\beta \alpha} x_{\alpha}=\Pi_{\beta \alpha} \Pi_{\alpha \delta} x_{\delta}=\Pi_{\beta \delta} x_{\delta}$ $=x_{\beta}$, and $x=\left\{x_{\alpha}\right\}=\bigvee_{\sigma} x^{\sigma} \in B^{i}$, and dually for $\Lambda_{\sigma} x^{\sigma}$.

3. The representation theorem. Let $B$ be a Boolean algebra. For $y \in B$, the principal ideal $(y)$ is the set of all $x \in B$ such that $x \leqq y$. For $x \leqq y, x, y \in B$, let $\phi_{x y}$ be a mapping with domain $(y)$ into $(x) \subset(y)$ such that for $z \in(y), \phi_{x y} z=z \bigcap x \in(x)$. It is trivial to show that $\phi_{x y}$ is a homomorphism of $(y)$ onto $(x)$ which preserves all (not only finite) '.u.b.'s and g.l.b.'s. From $x \leqq y \leqq z, x, y, z \in B$, we have $(x) \subset(y) \subset(z)$ 
and $\phi_{x z}=\phi_{x y} \phi_{y z}$. Thus, the set of Boolean algebras $\{(x)\}, x \in B$, is an inverse system. Call its inverse limit $C$. Clearly, $C \cong B$; for, if $(I)$ is the principal ideal generated by the supremum $I$ of $B$, then $(I)=B$. But $(I)$ is maximum in the set of sets $\{(x)\}$ under the ordering relation of inclusion. $C$ has a projection onto the component $(I)$, and it is easily seen that this projection is the required isomorphism.

Let $P$ be a set consisting of all finite-membered subsets $P_{\alpha}$ of $B$, where each $P_{\alpha}$ is a directed set under the order relation $\leqq$ of $B . P$ is directed by inclusion. For, given $P_{\alpha}$ and $P_{\beta} \in \mathcal{P}$, construct $P_{\gamma} \in \mathcal{P}$ as the set sum of $P_{\alpha}, P_{\beta}$, and $I$, the supremum of $B$. Then $P_{\alpha}, P_{\beta} \subset P_{\gamma}$. Since each $P_{\alpha}$ is finite, there exists a greatest element $x_{\alpha} \in P_{\alpha}$. Let $B_{\alpha}$ be the inverse limit algebra of the ideals $\left(y_{\alpha}\right)$ which are generated by the elements $y_{\alpha} \in P_{\alpha}$. Then $B_{\alpha} \cong\left(x_{\alpha}\right)$. Suppose that $P_{\alpha} \subset P_{\beta}$ with maximum elements $x_{\alpha}$ and $x_{\beta}$, respectively. Let $\left\{y_{\beta}^{t}\right\}$ be an element of $B_{\beta}$, where $t \in P_{\beta}$. Construct $\left\{y_{\alpha}^{t}\right\}$ in $B_{\alpha}^{*}$, the cardinal product of the factors of $B_{\alpha}$, by putting $y_{\alpha}^{t}=y_{\beta}^{t}$ for every $t \in P_{\alpha} .\left\{y_{\beta}^{t}\right\} \in B_{\alpha}$, since $x_{\alpha} \leqq x_{\beta}$, where $B_{\alpha} \cong\left(x_{\alpha}\right), B_{\beta} \cong\left(x_{\beta}\right)$. Define $\Pi_{\alpha \beta}$, a mapping from $B_{\beta}$ into $B_{\alpha}$, by $\Pi_{\alpha \beta}\left\{y_{\beta}^{z}\right\}=\left\{y_{\alpha}^{z}\right\}$. It is easy to show that $\Pi_{\alpha \beta}$ is a lattice homomorphism of $B_{\beta}$ onto $B_{\alpha}$ and that if $P_{\alpha} \subset P_{\beta} \subset P_{\gamma}$, then $\Pi_{\alpha \gamma}=\Pi_{\alpha \beta} \Pi_{\beta \gamma}$.

Let $D$ be the inverse limit algebra of the $B_{\alpha}$ 's. Consider those $P_{\alpha}$, and associate $B_{\alpha}$, for which $I \in P_{\alpha}$, where $I$ is the supremum of $B$. The set $S$ of such $P_{\alpha}$ is nonempty and cofinal in the set of all $P_{\alpha}$ as ordered by inclusion. $P_{\alpha} \in S$ implies $B_{\alpha} \cong B$. Note that there is no maximum element in $S$ (or in the set $P$ of all $P_{\alpha}$ ) if $B$ is infinite. Let $\Pi_{\alpha}$ be the projection of $D$ into a factor $B_{\alpha}$ associated with a $P_{\alpha} \in S$. It is routine to show that $\Pi_{\alpha}$ is onto $B_{\alpha}$ and that $\Pi_{\alpha}$ is an isomorphism. Since $B_{\alpha} \cong B$, then $D \cong B$. We have proved the following theorem.

Theorem 2. Let $B$ be an infinite Boolean algebra. $B$ is isomorphic to the inverse limit of an inverse system of Boolean algebras. Each algebra in this system is isomorphic to a principal ideal of $B$, and there is no final algebra in the system. Other than the algebra (0), each such ideal occurs with repetitions, and each principal ideal of $B$ appears. Under the ordering relation of the factors of the inverse system each factor is covered by an algebra isomorphic to $B$.

4. The direct limit. Let $A$ be a set partly ordered by $\leqq$ and directed. Let $\left\{B_{\alpha}\right\}$ be a set of Boolean algebras indexed by A. For each inequality $\beta \leqq \alpha, \alpha, \beta \in \mathrm{A}$, suppose that there exists a lattice homomorphism $\phi_{\beta \alpha}$ of $B_{\beta}$ into $B_{\alpha}$ in such a way that (1) $\phi_{\beta \alpha} I_{\beta}=I_{\alpha}$ and $\phi_{\beta \alpha} 0_{\beta}=0_{\alpha}$; and (2) $\gamma \leqq \beta \leqq \alpha(\gamma, \beta, \alpha \in \mathrm{A})$ imply $\phi_{\gamma \alpha}=\phi_{\beta \alpha} \phi_{\gamma \beta} . \phi_{\alpha \alpha}$ is to be the identity mapping of $B_{\alpha}$ onto $B_{\alpha}$. We can define an equivalence 
relation on the ordered pairs $\left(\alpha, x_{\alpha}\right)\left(\alpha \in \mathrm{A}, x_{\alpha} \in B_{\alpha}\right)$ by writing $\left(\alpha, x_{\alpha}\right)$ $\sim\left(\beta, x_{\beta}\right)$ if there exists $\gamma \geqq \alpha, \beta$ with $\phi_{\alpha \gamma} x_{\alpha}=\phi_{\beta \gamma} x_{\beta}$. Let $B^{d}$ be the set of all equivalence classes of such ordered pairs. Such classes are denoted by $\left\{\left(\alpha, x_{\alpha}\right)\right\}$ and can be ordered in a natural way by writing $\left\{\left(\alpha, x_{\alpha}\right)\right\} \leqq\left\{\left(\beta, x_{\beta}\right)\right\}$ if there exist $\left(\alpha_{1}, x_{\alpha_{1}}\right) \sim\left(\alpha, x_{\alpha}\right)$ and $\left(\beta_{1}, x_{\beta_{1}}\right)$ $\sim\left(\beta, x_{\beta}\right)$ and a $\gamma \in \mathrm{A}, \gamma \geqq \alpha_{1}, \beta_{1}$, such that $\phi_{\alpha_{1} \gamma} x_{\alpha_{1}} \leqq \phi_{\beta_{1} \gamma} x_{\beta_{1}}$. It can be proved that the relation $\leqq$ does not depend on the particular representatives of the equivalence classes which are employed. That is, there exists $\gamma \geqq \alpha, \beta$ with $\phi_{\alpha \gamma} x_{\alpha} \leqq \phi_{\beta \gamma} x_{\beta}$. Under this ordering, $B^{d}$ is a Boolean algebra, the element $\left\{\left(\alpha, I_{\alpha}\right)\right\}$ is the supremum of $B^{d}$, and $\left\{\left(\alpha, 0_{\alpha}\right)\right\}$ is the infimum. An element $\left\{\left(\alpha, x_{\alpha}\right)\right\}$ has the complement $\left\{\left(\alpha, x_{\alpha}^{\prime}\right)\right\}$, where $x_{\alpha}^{\prime}$ is the complement of $x_{\alpha}$ in $B_{\alpha}$. This Boolean algebra may be called the direct limit algebra of the $B_{\alpha}$ and may be denoted by $B^{d}=\lim _{\rightarrow} B_{\alpha}$.

5. An example of a direct limit. Let $X$ be a compactum which is, in at least one way, a homogeneous simplicial polyhedron $\alpha$ of dimension $n$ with only a finite number of $n$-faces. For a given finite, $n$-homogeneous, simplicial polyhedral decomposition $\alpha$ of $X$, the set of all simplicial subpolyhedra of dimension $n$ forms a Boolean algebra $B_{\alpha}$ in an obvious fashion if one admits the null polyhedron as an $n$-homogeneous subpolyhedron of every such subpolyhedron of $X$. Write $\alpha \leqq \beta$ if $\beta$ is a refinement of $\alpha$. That is, every closed $n$ face of $\beta$ is included in a closed $n$-face of $\alpha$. For $x_{\alpha} \in B_{\alpha}$ and $\alpha \leqq \beta$, define $\phi_{\alpha \beta} x_{\alpha}=x_{\beta} \in B_{\beta}$ by taking $x_{\beta}$ to be that subpolyhedron of $\beta$, the point set of which is the set sum of all $n$-faces of $\beta$ which lie in some face of $x_{\alpha} . \phi_{\alpha \beta}$ is a lattice homomorphism of $B_{\alpha}$ into $B_{\beta}$ in such a way that the null set is carried onto the null set, and $\alpha$ is carried onto $\beta$, the respective suprema of $B_{\alpha}$ and $B_{\beta}$. Given $\alpha$ and $\beta$, there exists a common refinement $\gamma$, a polyhedral covering of $X$ of the given type. It turns out that if $\alpha \leqq \beta \leqq \gamma$, then $\phi_{\alpha \gamma}=\phi_{\beta \gamma} \phi_{\alpha \beta}$. Therefore, the $B_{\alpha}$ with mappings $\phi_{\alpha \beta}$ are a direct system of Boolean algebras. The limit algebra $B^{d}$ is the algebra of all polyhedra which are subsets of $X$ and is an atomless Boolean algebra. (For instance, use barycentric subdivision to obtain a refinement of any given element.)

\section{Ideals.}

Theorem 3. Let $B^{d}=\lim _{\rightarrow} B_{\alpha}$. (1) For a given ideal $J$ in $B^{d}$, the set of all $x_{\alpha} \in B_{\alpha}$ for which $\left\{\left(\alpha, x_{\alpha}\right)\right\} \in J$ is an ideal $J_{\alpha}$ in $B_{\alpha}$. Let the $\phi_{\alpha \beta}$ be onto mappings. (2) For given $x \in J$ and $\alpha \in \mathrm{A}$, there exists $x_{\alpha} \in J_{\alpha}$ with $\left\{\left(\alpha, x_{\alpha}\right)\right\}=x$ (so that the $J_{\alpha}$ are nonvoid and are "representative" of $J$ ). (3) For $\alpha \leqq \beta, \phi_{\alpha \beta} J_{\alpha}=J_{\beta}$ and $\phi_{\alpha \beta}^{-1} J_{\beta}=J_{\alpha}$. (4) $J=\lim . J_{\alpha}$ with suitably restricted mappings $\phi_{\alpha \beta}$. 
Proof. (1), (2), and (3) are obvious. (4) Let $M$ be the set of all $\left\{\left(\alpha, x_{\alpha}\right)\right\} \in \lim _{\rightarrow} B_{\alpha}$ for which there exist representatives $\left(\alpha, x_{\alpha}\right)$ with $x_{\alpha} \in J_{\alpha}$. If $\left(\beta, x_{\beta}\right) \sim\left(\alpha, x_{\alpha}\right)$, there exists $\gamma \geqq \alpha, \beta$ with $\phi_{\alpha \gamma} x_{\alpha}$ $=\phi_{\beta \gamma} x_{\beta}$, and $\phi_{\beta \gamma} x_{\beta} \in J_{\gamma}, x_{\beta} \in J_{\beta}$, by (3). It is clear that $M=J$, but $M=\lim \rightarrow J_{\alpha}$ with the mappings $\phi_{\alpha \beta}$ restricted to the $J_{\alpha}$.

TheOREM 4. Let $B^{d}=\lim _{\rightarrow} B_{\alpha}$, and let the $\phi_{\alpha \beta}$ be onto mappings. For given $m \in B^{d}$, let $M_{\alpha}$ be the set of all $m_{\alpha} \in B_{\alpha}$ such that $\left\{\left(\alpha, m_{\alpha}\right)\right\}$ $=m$. (1) For $\alpha \leqq \beta, \phi_{\alpha \beta} M_{\alpha}=M_{\beta}$ and $\phi_{\alpha \beta}^{-1} M_{\beta}=M_{\alpha}$. (2) Suppose that there exists a selection function $f$ on $\mathbf{A}$ into the $M_{\alpha}$ such that $f(\alpha)=m_{\alpha}$ $\in M_{\alpha}$ in such a way that $\alpha \leqq \beta$ implies $\phi_{\alpha \beta} m_{\alpha}=m_{\beta}$. Then for the principal ideals $\left(m_{\alpha}\right)$ of $B_{\alpha}$ with suitably restricted mappings $\phi_{\alpha \beta}, \lim \rightarrow\left(m_{\alpha}\right)$ exists and equals $(m)$, the principal ideal in $B^{d}$ with supremum $m$. (3) Let the $B_{\alpha}$ be complete. Then the completions $(m)_{\alpha}^{-}$of the $(m)_{\alpha}$ are principal ideals in $B_{\alpha}$, and $(m)_{\alpha}^{-}=\left(\mathrm{V}_{m_{\alpha}}\right)$, where the 1.u.b. is taken over all $m_{\alpha} \in M_{\alpha}$. (4) Let the $B_{\alpha}$ be complete, and let the $\phi_{\alpha \beta}$ preserve all least upper bounds. Then $\phi_{\alpha \beta}(m)_{\alpha}^{-}=(m)_{\beta}^{-}$.

Proof. (1) is obvious. (2) $\phi_{\alpha \beta}$ restricted to $\left(m_{\alpha}\right)$ is into $\left(m_{\beta}\right)$. If $x_{\beta} \leqq m_{\beta}$, there exists $x_{\alpha}^{*} \in B_{\alpha}$ such that $\phi_{\alpha \beta} x_{\alpha}^{*}=x_{\beta}$. Let $x_{\alpha}=x_{\alpha}^{*} \cap m_{\alpha}$. Then $\phi_{\alpha \beta} x_{\alpha}=x_{\beta} \cap m_{\beta}=x_{\beta}$, so that $\phi_{\alpha \beta}$ is onto $\left(m_{\beta}\right)$. From this, $N$ $=\lim _{\rightarrow}\left(m_{\alpha}\right)$ exists and is a subset of $(m)$. For $\left\{\left(\alpha, y_{\alpha}\right)\right\} \in(m)$, $n_{\alpha}=y_{\alpha} \cup m_{\alpha} \in M_{\alpha}$. There exists $\gamma \in A, \gamma \geqq \alpha$, such that $\phi_{\alpha \gamma} n_{\alpha}=\phi_{\alpha \gamma} m_{\alpha}$. Then $\left\{\left(\alpha, y_{\alpha}\right)\right\}=\left\{\left(\gamma, \phi_{\alpha \gamma} y_{\alpha}\right)\right\}$. But $\phi_{\alpha \gamma} y_{\alpha} \leqq \phi_{\alpha \gamma} m_{\alpha}=m_{\gamma}$. Hence $\left\{\left(\alpha, y_{\alpha}\right)\right\} \in N$.

(3) $V m_{\alpha}$ exists, by the completeness of $B_{\alpha}$, and lies in $(m)_{\alpha}^{-}$. If $\left\{\left(\alpha, x_{\alpha}\right)\right\} \in(m)$, there exists $n_{\alpha} \in M_{\alpha}$ with $x_{\alpha} \leqq n_{\alpha}$, as we see from the proof of (2). Hence $\mathrm{V} x_{\alpha} \leqq \mathrm{V} m_{\alpha}$, where the first 1.u.b. is taken over all $x_{\alpha} \in(m)_{\alpha}$, and the second is taken over all $m_{\alpha} \in M_{\alpha}$. Hence $\mathrm{V} x_{\alpha}=\mathrm{V}_{m_{\alpha}}$, and the latter is the generator of the principal ideal $(m)_{\alpha}^{-}$in $B_{\alpha}$.

(4) If $x_{\alpha} \in(m)_{\alpha}^{-}$, then $x_{\alpha} \leqq \mathrm{V}_{m_{\alpha}}, \phi_{\alpha \beta} x_{\alpha} \leqq \mathrm{V}_{\phi_{\alpha \beta}} m_{\alpha}=\mathrm{V}_{m_{\beta}}$, so that $\alpha \leqq \beta$ implies $x_{\beta}=\phi_{\alpha \beta} x_{\alpha} \in(m)_{\beta}^{-}$. For $x_{\beta} \in(m)_{\beta}^{-}, x_{\beta} \leqq \bigvee_{\mu} m_{\beta}^{\mu}, m_{\beta}^{\mu} \in M_{\beta}$. There exist $x_{\beta}^{\mu} \leqq m_{\beta}^{\mu}$ such that $x_{\beta}=\bigvee_{\mu} x_{\beta}^{\mu}$, and there exist $x_{\alpha}^{\mu}$ such that $\phi_{\alpha \beta} x_{\alpha}^{\mu}=x_{\beta}^{\mu}$. Hence $x_{\alpha}^{\mu} \in(m)_{\alpha}$, and $\bigvee_{\mu} x_{\alpha}^{\mu}=x_{\alpha} \in(m)_{\alpha}^{-}$, with $\phi_{\alpha \beta} x_{\alpha}=\bigvee_{\mu} \phi_{\alpha \beta} x_{\alpha}^{\mu}$ $=\mathrm{V}_{\mu} x_{\beta}^{\mu}=x_{\beta}$.

Theorem 5. Let $B^{d}=\lim _{\rightarrow} B_{\alpha}$, and let the $\phi_{\alpha \beta}$ be onto mappings. Then the Boolean algebras $B_{\alpha} / J_{\alpha}$, for fixed ideal $J$ in $B^{d}$ and suitable induced mappings $\phi_{\alpha \beta}^{-}$, form a direct system, the direct limit of which is isomorphic to $B^{d} / J$.

Proof. For $\alpha \leqq \beta, x_{\alpha}^{-} \in B_{\alpha} / J_{\alpha}$, choose an antecedent $x_{\alpha} \in B_{\alpha}$ of $x_{\alpha}^{-}$. Let $\phi_{\alpha \beta}^{-} x_{\alpha}^{-}$be the coset of $J_{\beta}$ in $B_{\beta}$ with representative $\phi_{\alpha \beta} x_{\alpha}$. The mapping $\phi_{\alpha \beta}^{-}$of $B_{\alpha} / J_{\alpha}$ into $B_{\beta} / J_{\beta}$ clearly does not depend upon the 
representatives employed and is onto $B_{\beta} / J_{\beta}$. For $\alpha \leqq \beta \leqq \gamma, \phi_{\alpha \gamma}^{-} x_{\alpha}^{-}$ $=\left(\phi_{\alpha \gamma} x_{\alpha}\right)^{-}=\left(\phi_{\beta \gamma} \phi_{\alpha \beta} x_{\alpha}\right)^{-}=\phi_{\beta \gamma}^{-}\left(\phi_{\alpha \beta} x_{\alpha}\right)^{-}=\phi_{\beta \gamma}^{-} \phi_{\alpha \beta}^{-} x_{\alpha}^{-}$. The direct system of the $B_{\alpha} / J_{\alpha}$ thus exists, and its direct limit will be denoted by $D$. For $x=\left\{\left(\alpha, x_{\alpha}^{-}\right)\right\} \in D$, and for given $\alpha, \beta \in \mathrm{A}$, choose antecedents $x_{\alpha}$, $x_{\beta}$, where $\left(\beta, x_{\beta}^{-}\right) \sim\left(\alpha, x_{\alpha}^{-}\right)$. There exists $\gamma \geqq \alpha, \beta$ such that $\phi_{\alpha \gamma}^{-} x_{\alpha}^{-}$ $=\phi_{\beta \gamma}^{-} x_{\beta}^{-}$. That is, $\left(\phi_{\alpha \gamma} x_{\alpha}\right)^{-}=\left(\phi_{\beta \gamma} x_{\beta}\right)^{-}$. Computing the symmetric difference in $B^{d}=\lim _{\rightarrow} B_{\alpha}$, we have $\left\{\left(\alpha, x_{\alpha}\right)\right\}-\left\{\left(\beta, x_{\beta}\right)\right\}=\left\{\left(\gamma, \phi_{\alpha \gamma} x_{\alpha}\right.\right.$ $\left.\left.-\phi_{\beta \gamma} x_{\beta}\right)\right\}$. But $\phi_{\alpha \gamma} x_{\alpha}-\phi_{\beta \gamma} x_{\beta} \in J_{\gamma}$, since $\left(\phi_{\alpha \gamma} x_{\alpha}\right)^{-}=\left(\phi_{\beta \gamma} x_{\beta}\right)^{-}$. Hence the symmetric difference in $B^{d}$ is in $J$. There is thus defined a singlevalued map $\Phi$ on $D$ into $B^{d} / J$ given by $\Phi\left\{\left(\alpha, x_{\alpha}^{-}\right)\right\}=\left\{\left(\alpha, x_{\alpha}\right)\right\}-$. $\Phi$ is onto, since for each $\left\{\left(\alpha, x_{\alpha}\right)\right\}-\in B^{d} / J, \Phi\left\{\left(\alpha, x_{\alpha}^{-}\right)\right\}=\left\{\left(\alpha, x_{\alpha}\right)\right\}-$. If $\Phi\left\{\left(\alpha, x_{\alpha}^{-}\right)\right\}=\Phi\left\{\left(\beta, y_{\beta}^{-}\right)\right\}$, then $\left\{\left(\alpha, x_{\alpha}\right)\right\}-=\left\{\left(\beta, y_{\beta}\right)\right\}-$, and there exists $\gamma \geqq \alpha, \beta$ with $\phi_{\alpha \gamma} x_{\alpha}-\phi_{\beta \gamma} y_{\beta} \in J_{\gamma}$. Thus, $\left\{\left(\alpha, x_{\alpha}^{-}\right)\right\}=\left\{\left(\gamma, \phi_{\alpha \gamma}^{-} x_{\alpha}^{-}\right)\right\}$ $=\left\{\left(\gamma,\left(\phi_{\alpha \gamma} x_{\alpha}\right)^{-}\right)\right\}=\left\{\left(\gamma,\left(\phi_{\beta \gamma} y_{\beta}\right)^{-}\right)\right\}=\left\{\left(\gamma, \phi_{\beta \gamma}^{-} y_{\beta}^{-}\right)\right\}=\left\{\left(\beta, y_{\beta}^{-}\right)\right\}$. This shows that $\Phi^{-1}$ is single-valued, hence one-to-one on $D$ onto $B^{d} / J$. If $\left.\left\{\left(\alpha, x_{\alpha}\right)\right\}^{-\geqq} \geqq\left(\beta, y_{\beta}\right)\right\}^{-}=\left\{\left(\alpha, y_{\alpha}\right)\right\}^{-}$in $B^{d} / J$, there exist $\left\{\left(\alpha, x_{\alpha}\right)\right\} \in\left\{\left(\alpha, x_{\alpha}\right)\right\}-,\left\{\left(\alpha, y_{\alpha}\right)\right\} \in\left\{\left(\alpha, y_{\alpha}\right)\right\}-$ with $\left\{\left(\alpha, x_{\alpha}\right)\right\}$ $\geqq\left\{\left(\alpha, y_{\alpha}\right)\right\}$ in $B^{d}$. There exists $\alpha \in \mathrm{A}$ with $x_{\alpha} \geqq y_{\alpha}$. Hence $x_{\alpha}^{-} \geqq y_{\alpha}^{-}$, and $\left\{\left(\alpha, x_{\alpha}^{-}\right)\right\} \geqq\left\{\left(\alpha, y_{\alpha}^{-}\right)\right\} . \Phi^{-1}$ thus preserves $\geqq$. As for $\Phi,\left\{\left(\alpha, x_{\alpha}^{-}\right)\right\}$ $\geqq\left\{\left(\alpha, y_{\alpha}^{-}\right)\right\}=\left\{\left(\beta, y_{\beta}^{-}\right)\right\}$in $D$ implies the existence of $\alpha$ such that $x_{\alpha}^{-} \geqq y_{\alpha}^{-}$in $B_{\alpha} / J_{\alpha}$. There exist $x_{\alpha} \in B_{\alpha}, y_{\alpha} \in B_{\alpha}$ with $x_{\alpha} \geqq y_{\alpha} ;$ and $\left\{\left(\alpha, x_{\alpha}\right)\right\} \geqq\left\{\left(\alpha, y_{\alpha}\right)\right\}$ in $B^{d}$. Also, $\left\{\left(\alpha, x_{\alpha}\right)\right\}-\geqq\left\{\left(\alpha, y_{\alpha}\right)\right\}^{-}$in $B^{d} / J$. Hence $\Phi\left\{\left(\alpha, x_{\alpha}^{-}\right)\right\} \geqq \Phi\left\{\left(\beta, y_{\beta}^{-}\right)\right\}$. This proves the theorem.

7. Ideal decomposition. To each $x_{\alpha} \in B_{\alpha}$ let correspond $\left\{\left(\alpha, x_{\alpha}\right)\right\}$ $\in B^{d}$. This determines a mapping $\phi_{\alpha}$ of $B_{\alpha}$ into $B^{d}$, a lattice homomorphism with kernel $\mathfrak{l}_{\alpha} \cdot \phi_{\alpha}$ is onto $B^{d}$; for, $\left\{\left(\beta, x_{\beta}\right)\right\} \in B^{d}, \gamma \geqq \alpha, \beta$ imply the existence of $x_{\alpha} \in B_{\alpha}$ with $\phi_{\alpha \gamma} x_{\alpha}=\phi_{\beta \gamma} x_{\beta}$, so that $\left\{\left(\alpha, x_{\alpha}\right)\right\}$ $=\left\{\left(\gamma, \phi_{\beta \gamma} x_{\beta}\right)\right\}=\left\{\left(\beta, x_{\beta}\right)\right\}$.

Let $J$ and $K$ be ideals in a Boolean algebra $B$. Suppose that (1) $x \in J, K$ imply $x=0$ and that (2) to each $x \in B$, there exist $y \in J$, $z \in K$ with $x=y \cup z$. It is easily seen that $J$ and $K$ are principal ideals. $B$ is said to be the cardinal product [1] of $J$ and $K$. In general, let $\Sigma$ be an index class, and let $\left\{J^{\sigma}\right\}, \sigma \in \Sigma$, be a set of ideals of $B$, indexed by $\Sigma$, with the properties (1) for $x^{\sigma} \in J^{\sigma}$ and for every existing $\bigvee_{\tau \neq \sigma} x^{\tau}, x^{\tau} \in J^{\tau}, \tau \neq \sigma$, one has $x^{\sigma} \cap \bigvee_{\tau \neq \sigma} x^{\tau}=0$; (2) to each $x \in B$ correspond $x^{\sigma} \in J^{\sigma}, \sigma \in \Sigma$, such that $x=\mathrm{V}_{\sigma} x^{\sigma}$. $B$ is said to be the cardinal product of the (principal) ideals $J^{\sigma}$.

TheOREM 6. Let the $\phi_{\alpha \beta}$ be onto mappings, where $B^{d}=\lim _{\rightarrow} B_{\alpha} . B^{d}$ is the cardinal product of ideals $J$ and $K$ if, and only if, (a) for each $\alpha \in \mathrm{A}$ and each $x_{\alpha} \in B_{\alpha}$, there exist $y_{\alpha} \in J_{\alpha}, z_{\alpha} \in K_{\alpha}$, and $k_{\alpha} \in \mathfrak{l}_{\alpha}$ with $x_{\alpha}-\left(y_{\alpha} \cup z_{\alpha}\right)=k_{\alpha} ;$ and (b) $x_{\sigma} \in J_{\alpha}, K_{\alpha}$ imply $x_{\alpha} \in \mathfrak{f}_{\alpha}$, for every $\alpha \in \mathrm{A}$. 
Proof. If $B^{d}=J K$, then $\left\{\left(\alpha, x_{\alpha}\right)\right\}=\left\{\left(\alpha, y_{\alpha}\right)\right\} \cup\left\{\left(\alpha, z_{\alpha}\right)\right\}, y_{\alpha} \in J_{\alpha}$, $z_{\alpha} \in K_{\alpha} . x_{\alpha}-\left(y_{\alpha} \cup z_{\alpha}\right) \in \mathfrak{f}_{\alpha}$. As for (b), $x_{\alpha} \in J_{\alpha}, K_{\alpha}$ imply $\left\{\left(\alpha, x_{\alpha}\right)\right\}$ $\in J, K$. Hence $\left\{\left(\alpha, x_{\alpha}\right)\right\}=0$, and $x_{\alpha} \in \mathfrak{l}_{\alpha}$. Conversely, given (a) and (b), for $\left\{\left(\alpha, x_{\alpha}\right)\right\} \in B^{d}$, there exist $y_{\alpha} \in J_{\alpha}, z_{\alpha} \in K_{\alpha}$, and $k_{\alpha} \in \mathfrak{f}_{\alpha}$ with $x_{\alpha}-\left(y_{\alpha} \cup z_{\alpha}\right)=k_{\alpha}$. This implies $\left\{\left(\alpha, x_{\alpha}\right)\right\}=\left\{\left(\alpha, y_{\alpha}\right)\right\} \cup\left\{\left(\alpha, z_{\alpha}\right)\right\}$ so that $J$ and $K$ generate $B^{d}$. If $\left\{\left(\alpha, x_{\alpha}\right)\right\} \in J, K$, then $x_{\alpha} \in J_{\alpha}, K_{\alpha}$ so that $x_{\alpha} \in \mathfrak{l}_{\alpha}$ and $\left\{\left(\alpha, x_{\alpha}\right)\right\}=0$.

CoROLlaRY. Under the conditions of the theorem, if each $B_{\alpha}$ is the cardinal product of $J_{\alpha}$ and $K_{\alpha}$, then $B^{d}$ is the cardinal product of $J$ and $K$.

Theorem 7. Let $B^{d}=\lim _{\rightarrow} B_{\alpha}$ where the $B_{\alpha}$ are complete. Let the $\phi_{\alpha \beta}$ be onto mappings. Suppose that $B^{d}$ has the property that for every $\alpha$, $\mathrm{V}_{\sigma}\left\{\left(\alpha, x_{\alpha}^{\sigma}\right)\right\}$ exists and equals $\left\{\left(\alpha, \mathrm{V}_{\sigma} x_{\alpha}^{\sigma}\right)\right\}$. Then $B^{d}$ is the cardinal product of ideals $\left\{J^{\sigma}\right\}$ if, and only if, $\left(\mathrm{a}^{\prime}\right)$ for each $\alpha \in \mathrm{A}$ and $x_{\alpha} \in B_{\alpha}$, there exist $y_{\alpha}^{\sigma} \in J_{\alpha}^{\sigma}$ and $k_{\alpha} \in \mathfrak{l}_{\alpha}$ with $x_{\alpha}-\bigvee_{\sigma} y_{a}^{\sigma}=k_{\alpha} ;$ and $\left(\mathrm{b}^{\prime}\right) x_{\alpha}^{\sigma} \in J_{\alpha}^{\sigma}$, $x_{\alpha}^{\tau} \in J_{\alpha}^{\tau}, \tau \neq \sigma$, yield $x_{\alpha}^{\sigma} \cap \bigvee_{\tau \neq \sigma} x_{\alpha}^{\tau} \in \mathfrak{l}_{\alpha}$.

The proof is like that of Theorem 6 .

CoRollary. If, under the conditions of Theorem 7, each $B_{\alpha}$ is the cardinal product of the $J_{\alpha}^{\sigma}$, then $B^{d}$ is the cardinal product of the $J^{\sigma}$.

8. Ideals in the inverse limit. For the inverse limits, the results of $\S \S 6$ and 7 have appropriate counterparts. Since the proofs are straightforward or are similar to those of $\$ \$ 6$ and 7, only the results will be stated. Given $B^{i}=\lim _{+}\left(B_{\alpha}, \Pi_{\beta \alpha}\right)$, the set of all $x_{\alpha} \in B_{\alpha}$ for which there exists $x \in B^{i}$ with $\alpha$-component $x_{\alpha}$ is a subalgebra of $B_{\alpha}$. $\Pi_{\beta \alpha}$ restricted to this subalgebra is on to the corresponding subalgebra of $B_{\beta}$; and if we replace each $B_{\alpha}$ by its corresponding subalgebra (as defined here), then $B^{i}$ is not changed. For the rest of this section, we assume this replacement to have been made.

For an ideal $J$ in $B^{i}$, let $J_{\alpha}$ be the set of all $x_{\alpha} \in B_{\alpha}$ for which $x_{\alpha}$ is the $\alpha$-component of some $x \in J$. Then $J_{\alpha}$ is an ideal in $B_{\alpha} . \Pi_{\beta \alpha} J_{\alpha}=J_{\beta}$, and if the kernel of $\Pi_{\beta \alpha}$ is included in $J_{\alpha}$, then $\Pi_{\beta \alpha}^{-1} J_{\beta}=J_{\alpha}$. Corresponding to each ideal $J$ in $B^{i}$, there is an ideal $J^{\sharp}=\lim _{+}\left(J_{\alpha}, \Pi_{\beta \alpha}\right)$ in $B^{i}$ such that $J \subset J^{*}$ and such that $J_{\alpha}^{*}=J_{\alpha}$ for every $\alpha \in \mathrm{A}$. If $J$ is prime, then $J^{\sharp}=J$ or $J^{\sharp}=B^{i}$. If some $J_{\alpha}$ is proper for $J$ prime, then $J=J^{*}$. If $J$ is prime then $J_{\alpha}$ is prime or $J_{\alpha}=B_{\alpha}$ for a given index $\alpha$. Likewise, if $J$ is principal, then $J^{t}=J$.

Let $x J$ represent that coset of $J$ which contains the element $x$. For $J$ an ideal in $B^{i}$, define a mapping $\Pi_{\beta \alpha}^{*}$ of $B_{\alpha} / J_{\alpha}$ into $B_{\beta} / J_{\beta}$ $(\beta \leqq \alpha)$ by $\Pi_{\beta \alpha}^{*}\left(x_{\alpha} J_{\alpha}\right)=\left(\Pi_{\beta \alpha} x_{\alpha}\right) J_{\beta}$. $\Pi_{\beta \alpha}^{*}$ turns out to be a homomorphism of $B_{\alpha} / J_{\alpha}$ onto $B_{\beta} / J_{\beta}$ and is independent of the representa- 
tive $x_{\alpha}$ of the coset $x_{\alpha} J_{\alpha}$. If $J^{\#}=J$, then $B^{i} / J$ is isomorphic to a subalgebra of $\lim _{+}\left(B_{\alpha} / J_{\alpha}, \Pi_{\beta \alpha}^{*}\right)$.

$B^{i}=J^{\#} K^{\#}$, the cardinal product of $J^{\#}$ and $K^{\#}$, if, and only if, $B_{\alpha}=J_{\alpha} K_{\alpha}$ for every $\alpha \in \mathrm{A} . B^{i}=J K$ implies the same statement as above on the $B_{\alpha}$ 's. If the $B_{\alpha}$ are complete and if the $\Pi_{\beta \alpha}$ are complete mappings, then $B^{i}$ is the cardinal product of a collection of ideals $\left\{\left(J^{\sigma}\right)^{\#}\right\}$, a collection indexed by a set $\Sigma$, if and only if each $B_{\alpha}$ is the cardinal product of the collection $\left\{J_{\alpha}^{\sigma}\right\}$. If $B^{i}$ is the cardinal product of the collection $\left\{J^{\sigma}\right\}$, then the same statement as above on the $B_{\alpha}$ 's holds.

9. ${ }^{2}$ The Stone spaces. Let $B_{\alpha}$ be a Boolean algebra, and let $\Omega_{\alpha}$ be the collection of prime ideals of $B_{\alpha} . \Omega_{\alpha}$ is topologized by letting the neighborhoods of a base be all $\mathfrak{D}(x)$, where $\mathfrak{D}(x)$ is the set of all prime ideals of $B_{\alpha}$ which contain the element $x$ of $B_{\alpha}$. (See, for instance, [4].) Let $T_{\beta \alpha}$ be a homomorphism on a Boolean algebra $B_{\alpha}$ onto a subalgebra of a Boolean algebra $B_{\beta}$. For any prime ideal $M_{\beta}$ in $B_{\beta}$, the complete inverse image $T_{\beta \alpha}^{-1} M_{\beta}$ is a prime ideal in $B_{\alpha}$. Define a mapping $T_{\alpha \beta}^{*}$ on the Stone space $\Omega_{\beta}$ into the Stone space $\Omega_{\alpha}$ by $T_{\alpha \beta}^{*} M_{\beta}$ $=T_{\beta \alpha}^{-1} M_{\beta}$. For $x \in B_{\alpha}$, let $T_{\beta \alpha} x=y \in B_{\beta}$. A brief check shows that $\mathfrak{D}(y)$ $=T_{\alpha \beta}^{*-1} \mathfrak{D}(x)$, where $\mathfrak{D}(x)$ is a base neighborhood in $\Omega_{\alpha}, \mathfrak{D}(y)$ in $\Omega_{\beta}$. $T_{\alpha \beta}^{*}$ is thus continuous in a strong sense. We note that $x_{\alpha} \in T_{\alpha \beta}^{*} M_{\beta}$ if, and only if, $T_{\beta \alpha} x_{\alpha} \in M_{\beta}$.

Now let $R_{\alpha \beta}$ be a function continuous-in-the-strong-sense from $\Omega_{\beta}$ into $\Omega_{\alpha}$. That is, to $\mathfrak{D}(x), x \in B_{\alpha}$, corresponds $\mathfrak{D}(y), y \in B_{\beta}$, with $\mathfrak{D}(y)=R_{\alpha \beta}^{-1} \mathfrak{D}(x)$. Define a mapping $R_{\beta \alpha}^{\sharp}$ on $B_{\alpha}$ into $B_{\beta}$ as follows. For $x \neq 0_{\alpha}, x \in B_{\alpha}$, note that $R_{\alpha \beta}^{-1}\left[\mathfrak{D}\left(x^{\prime}\right)\right]$ is a neighborhood $\mathfrak{D}\left(y^{\prime}\right)$ in $\Omega_{\beta}$, $y \in B_{\beta}$. Let $R_{\beta \alpha}^{\#} x=y$. For $x=0_{\alpha}$, let $R_{\beta \alpha}^{*} 0_{\alpha}=0_{\beta}$. Using Stone's representation theorem [3], it is easy to show that $R_{\beta \alpha}^{\#}$ is a homomorphism on $B_{\alpha}$ onto a subalgebra of $B_{\beta}$. Also, $x_{\alpha} \in R_{\alpha \beta} M_{\beta}$ if, and only if, $R_{\beta \alpha}^{*} x_{\alpha} \in M_{\beta}$. Here, $M_{\beta}$ is a prime ideal of $B_{\beta}$.

From these definitions, we can show that $T_{\beta \alpha}^{* \#}=T_{\beta \alpha}$ and that $R_{\alpha \beta}^{H *}=R_{\alpha \beta}$. It follows that the homomorphisms of $B_{\alpha}$ onto subalgebras of $B_{\beta}$ are in one-to-one correspondence with the continuous-inthe-strong-sense mappings of $\Omega_{\beta}$ into $\Omega_{\alpha}$. By standard arguments, we can show that $T_{\alpha \beta}^{*}$ is onto $\Omega_{\alpha}$ if, and only if, $T_{\beta \alpha}$ is an isomorphism of $B_{\alpha}$ onto a subalgebra of $B_{\beta}$; that if $T_{\beta \alpha}$ is onto $B_{\beta}$, then $T_{\alpha \beta}^{*}$ is one-toone on $\Omega_{\beta}$ onto a subset of $\Omega_{\alpha}$; and that $T_{\alpha \beta}^{*}$ is one-to-one on $\Omega_{\beta}$ onto a subset of $\Omega_{\alpha}$ if, and only if, every prime ideal of the subalgebra $T_{\beta \alpha} B_{\alpha}$ can be extended to a prime ideal of $B_{\beta}$ in one and only one

2 Some of the statements of this section are due (with occasional modifications) to the referee, who suggested the investigation of this and of succeeding sections. 
way. (This extension is the smallest convex set in $B_{\beta}$ which contains the given prime ideal of $T_{\beta \alpha} B_{\alpha}$.)

10. The induced limits. Let $B_{\alpha}, B_{\beta}$, and $B_{\gamma}$ be Boolean algebras with appropriate homomorphisms $T_{\gamma \alpha}$ (on $B_{\alpha}$ onto a subalgebra of $B_{\gamma}$ ), and so forth. It follows quickly from the definitions that $T_{\gamma \alpha}$ $=T_{\gamma \beta} T_{\beta \alpha}$ if, and only if, $T_{\alpha \gamma}^{*}=T_{\alpha \beta}^{*} T_{\beta \gamma}^{*}$.

This shows that to each inverse limit $\lim _{\leftarrow}\left(B_{\alpha}, \Pi_{\beta \alpha}\right)$ of Boolean algebras there corresponds a direct limit $\lim _{\rightarrow}\left(\Omega_{\alpha}, \Pi_{\alpha \beta}^{*}\right)$ of the Stone spaces, where $\Pi_{\alpha \beta}^{*}$ is defined as in $\$ 9$; and to each direct limit $\lim _{\rightarrow}\left(B_{\alpha}, \phi_{\alpha \beta}\right)$ of Boolean algebras there corresponds an inverse limit $\lim _{\leftarrow}\left(\Omega_{\alpha}, \phi_{\beta \alpha}^{*}\right)$. Conversely, if the $\theta_{\beta \alpha}$ are continuous-in-the-strongsense, then $\lim _{\leftarrow}\left(\Omega_{\alpha}, \theta_{\beta \alpha}\right)$ induces $\lim _{\rightarrow}\left(B_{\alpha}, \theta_{\alpha \beta}^{\neq}\right)$, and likewise for $\lim _{\rightarrow}\left(\Omega_{\alpha}, \psi_{\alpha \beta}\right)$ and $\lim _{\leftarrow}\left(B_{\alpha}, \psi_{\beta \alpha}^{*}\right)$, where the $\psi_{\alpha \beta}$ are continuous in the strong sense.

Theorem 8. Let $S$ be the Stone space of $B^{i}=\lim _{\leftarrow}\left(B_{\alpha}, \Pi_{\beta \alpha}\right)$, where each $x_{\alpha} \in B_{\alpha}$ is the $\alpha$-component of some $x \in B^{i}$. Let $T=\lim _{\rightarrow}\left(\Omega_{\alpha}, \Pi_{\alpha \beta}^{*}\right)$ (where $\Omega_{\alpha}$ is the Stone space of $B_{\alpha}$ ). Then there exists a one-to-one map $\sigma$ on $T$ onto a closed subset of $S$.

Proof. By $\S 8$, all $\Pi_{\beta \alpha}$ are onto mappings. By $\S 9$, this makes the $\Pi_{\alpha \beta}^{*}$ one-to-one maps. Let $y$ be an element of $T$. For an index $\alpha$ and for prime ideals $M_{\alpha}^{1}$ and $M_{\alpha}^{2}$ in $B_{\alpha}$ suppose that $\left(\alpha, M_{\alpha}^{1}\right)$ and $\left(\alpha, M_{\alpha}^{2}\right)$ are both in $y$. Then there exists an index $\beta \geqq \alpha$ such that $\Pi_{\beta \alpha}^{*} M_{\alpha}^{1}$ $=\Pi_{\beta \alpha}^{*} M_{\alpha}^{2}$. Since $\Pi_{\beta \alpha}^{*}$ is one-to-one, $M_{\alpha}^{1}=M_{\alpha}^{2}$. Now define a subset $N(y)$ of $B^{i}$ by $N(y)=E\left[x ; x \in B^{i},\left(\alpha, M_{\alpha}\right) \in y\right.$ implies $\left.x_{\alpha} \in M_{\alpha}\right]$. 0 , the infimum of $B^{i}$, is in $N(y)$ so that $N(y)$ is nonvoid. It is trivial to show that $N(y)$ is a prime ideal in $B^{i}$. Define a mapping $\sigma$ on $T$ into $S$ by $\sigma(y)=N(y)$. By $\$ 8,[N(y)]_{\alpha}=M_{\alpha}$.

Suppose that $\sigma(y)=\sigma(z)=N, y, z \in T, N \in S$. There is an index $\alpha$ and a pair of prime ideals, $M_{\alpha}^{1}, M_{\alpha}^{2}$, in $B_{\alpha}$ such that $\left(\alpha, M_{\alpha}^{1}\right) \in y$, $\left(\alpha, M_{\alpha}^{2}\right) \in z$. But by the above, $M_{\alpha}^{1}=N_{\alpha}$ and $M_{\alpha}^{2}=N_{\alpha}$. Hence $y=z$, and $\sigma$ is one-to-one.

Let $M$ be a prime ideal of $B^{i}$. Suppose that $x \in M$ implies $x_{\alpha}=I_{\alpha}$ for no index $\alpha \in \mathrm{A}$. By $\S 8$, each $M_{\alpha}$ is a prime ideal in $B_{\alpha}$. For $\alpha \leqq \beta$, $\Pi_{\beta \alpha}^{*} M_{\alpha}=M_{\beta}$, so that $y=\left\{\left(\alpha, M_{\alpha}\right)\right\}$ is a point of $T . \sigma(y)=N(y)$ where $[N(y)]_{\alpha}=M_{\alpha}$. Since $N(y)$ is prime in $B^{i}$, $\$ 8$ shows that $N(y)=M$. We have thus determined the range of $\sigma$.

The complement of this range in $S$ consists of all those prime ideals of $B^{i}$ which contain elements of the form $x=\left\{x_{\alpha}\right\}$ where some $x_{\alpha}=I_{\alpha}$. Thus this complement is the set sum of open sets, ergo an open set; and the range of $\sigma$ is closed in $S$. 
CoROllaRY 1. Under the conditions of the theorem, let each $\Pi_{\beta \alpha}$ be an isomorphism of $B_{\alpha}$ onto $B_{\beta}$. Then range $\sigma=S$.

The proof is immediate.

CoRollary. 2. Under the conditions of the theorem, $T$ $=\lim _{\rightarrow}\left(\Omega_{\alpha}, \Pi_{\alpha \beta}^{*}\right)$ can be given the topology of a Stone space.

Proof. $\sigma$ is one-to-one on $T$ into a closed, hence compact, subset $C$ of $S$. $C$ has a relative topology from $S$. Since $S$ is totally disconnected, so is $C$ in the relative topology. Hence $C$ is a Stone space. Introducing the topology of $C$ into $T$ via $\sigma$, we make $T$ a Stone space.

We should note that no a priori topology is to be expected in $T=\lim _{\rightarrow}\left(\Omega_{\alpha}, \Pi_{\alpha \beta}^{*}\right)$, even though each Stone space $\Omega_{\alpha}$ has a topological structure and each $\Pi_{\alpha \beta}^{*}$ is continuous in-the-strong-sense. (See Hurewicz and Wallman, Dimension theory, Princeton, 1941, p. 133, footnote $\dagger$.)

TheOREM 9. Let $S$ be the Stone space of $B^{d}=\lim _{\rightarrow}\left(B_{\alpha}, \phi_{\alpha \beta}\right)$, and let $T=\lim _{\leftarrow}\left(\Omega_{\alpha}, \phi_{\beta \alpha}^{*}\right)$. Then $S$ and $T$ are homeomorphic.

Proof. A set of prime ideals $y=\left\{M_{\alpha}\right\}$, indexed by $\mathrm{A}$, is an element of $T$ if, and only if, $M_{\alpha} \in \Omega_{\alpha}$, and $\alpha \leqq \beta$ implies $\phi_{\beta \alpha}^{*} M_{\beta}=M_{\alpha}$ (that is, $\left.\phi_{\alpha \beta}^{-1} M_{\beta}=M_{\alpha}\right)$. Let $N(y)=E\left[x ; x \in B^{d}, x=\left\{\left(\alpha, x_{\alpha}\right)\right\}\right.$ where each such $\left.x_{\alpha} \in M_{\alpha}\right]$. A tedious but trivial proof shows that $N(y)$ is a prime ideal in $B^{d}$. Also the $\alpha$-component $[N(y)]_{\alpha}$ of $N(y)$ turns out to be $M_{\alpha}$.

Now let $\lambda$ be a mapping on $T$ into $S$ given by $\lambda(y)=N(y)$. If $\lambda\left\{M_{\alpha}^{(1)}\right\}=\lambda\left\{M_{\alpha}^{(2)}\right\}=N$, then $M_{\alpha}^{(1)}=N_{\alpha}=M_{\alpha}^{(2)}$ for every $\alpha \in \mathrm{A}$, and $\lambda$ is one-to-one. For a given prime ideal $N$ of $B^{d}$ it is immediate that $\alpha \leqq \beta$ implies $\phi_{\alpha \beta}^{-1} N_{\beta}=N_{\alpha}$, so that $y=\left\{N_{\alpha}\right\} \in T$. A brief check shows that $\lambda(y)=N$. Hence $\lambda$ is onto $S$.

In the Cartesian product $P_{\beta} \Omega_{\beta}$ of all the Stone spaces $\Omega_{\beta}$, we can pick out a basis for the standard topology as follows. We select a finite set of indices $\alpha(1), \alpha(2), \cdots, \alpha(n) \in \mathrm{A}$. Choose elements $x_{\alpha(1)}^{(1)}$ $\in B_{\alpha(1)}, x_{\alpha(2)}^{(2)} \in B_{\alpha(2)}, \cdots, x_{\alpha(n)}^{(n)} \in B_{\alpha(n)}$. Then form the Cartesian product $\mathfrak{D}^{*}$ of factors $\Omega_{\alpha}$ for $\alpha \in \mathrm{A}, \alpha \neq \alpha(i), \mathfrak{D}\left(x_{\alpha(i)}^{(i)}\right), i=1,2, \cdots, n$. (See $[2$, pp. $6-10]$.) Let $\mathfrak{D}$ be the relativization of $D^{*}$ in $T$. The set of all such $\mathfrak{D}$ forms a basis for the relative topology of $T$ in $P_{\beta} \Omega_{\beta}$. Set $x^{(i)}=\left\{\left(\alpha(i), x_{\alpha(i)}^{(i)}\right)\right\} \in B^{d}, i=1,2, \cdots, n$. Now $y=\left\{M_{\alpha}\right\} \in \mathcal{D}$ if, and only if, $x^{(i)} \in N(y), i=1,2, \cdots, n$. For, if $\left\{M_{\alpha}\right\} \in \mathcal{D}$ and if $\left\{\left(\alpha, z_{\alpha}\right)\right\}=\left\{\left(\alpha(i), x_{\alpha(i)}^{(i)}\right)\right\}$, then there exists an index $\gamma \geqq \alpha, \alpha(i)$ such that $\phi_{\alpha \gamma} z_{\alpha}=\phi_{\alpha(i) \gamma} x_{\alpha(i)}^{(i)} \in M_{\gamma}$, since $x_{\alpha(i)}^{(i)} \in M_{\alpha(i)}$. But $\phi_{\alpha \gamma}^{-1} M_{\gamma}=M_{\alpha}$. Hence $z_{\alpha} \in M_{\alpha}$. This shows that $x^{(i)}$ is in $N(y)$. The converse is obvi- 
ous. The above statement is equivalent to the fact that $\lambda(\mathfrak{D})$ $=\mathfrak{O}\left(x^{(i)}\right) \cap \mathfrak{O}\left(x^{(2)}\right) \cap \cdots \cap \mathfrak{}\left(x^{(n)}\right)$, an open set in $S$. Thus $\lambda^{-1}$ is contipuous.

Conversely, for $x \in B^{d}$, let 2 be that subset of $T$ for which $\lambda(Q)$ $=\mathfrak{D}(x)$. Choose $\alpha \in \mathrm{A}$ such that $x=\left\{\left(\alpha, x_{\alpha}\right)\right\}$. Then $y=\left\{M_{\alpha}\right\} \in \mathcal{Q}$ if, and only if, $x_{\alpha} \in M_{\alpha}$. Hence 2 is the relativization of a Cartesian product of factors $\Omega_{\beta}$ if $\beta \neq \alpha$, and $\supseteq\left(x_{\alpha}\right) .2$ is thus open, and $\lambda$ is continuous. This proves that $\lambda$ is indeed a homeomorphism.

\section{BIBLIOGRAPHY}

1. G. Birkhoff, Lattice theory, Amer. Math. Soc. Colloquium Publications, vol. 25, rev. ed., New York, 1949.

2. S. Lefschetz, Algebraic topology, Amer. Math. Soc. Colloquium Publications, vol. 27, New York, 1942.

3. R. Sikorski, On the representation of Boolean algebras as fields of sets, Fund. Math. vol. 35 (1948) pp. 247-258.

4. M. H. Stone, Applications of the theory of Boolean rings to general topology, Trans. Amer. Math. Soc. vol. 41 (1937) pp. 375-481.

Washington University in St. Louis

\section{INTEGRAL BASES AND VARIETIES MULTIPLY OF THE FIRST SPECIES}

H. T. MUHLY

1. The object of this note is to point out the connection between varieties multiply of the first species in the sense of Dubreil ${ }^{1}$ and those irreducible varieties $V$ which are such that the ring of homogeneous coordinates of the general point of $V$ admits an independent linear base over the subring generated by a suitably chosen set of independent variables.

2. Let $k$ be a field of characteristic zero and let $R$ $=k\left[X_{0}, X_{1}, \cdots, X_{n}\right]$ be the ring of polynomials in $n+1$ indeterminates over $k$. If $\mathfrak{a}$ is an $H$-ideal in $R$ with no irrelevant components, $\mathfrak{a}$ is said to be of the first species if there exists a linear form $L=\sum c_{i} X_{i}$, $c_{i} \in k$, which does not belong to any of the relevant prime ideals of $\mathfrak{a}$ and is such that $a+R \cdot L$ has no irrelevant components. It is known

Presented to the Society, November 25, 1950; received by the editors August 22, 1950.

${ }^{1} \mathrm{P}$. Dubreil, Quelques propriêtés des variêtês algêbriques se rattachant aux théories de l'algèbre moderne, Actualités Scientifiques et Industrielles, vol. 210, 1935. P. Dubreil, Sur la dimension des idéaux de polynomes, J. Math. Pures Appl. (9) vol. 15 (1936). These will be referred to as D1 and D2 respectively. 